\title{
CHROMOSOME REGION-SPECIFIC LIBRARIES FOR HUMAN GENOME ANALYSIS
}

\section{PROGRESS REPORT}

for Period September 1, 1991 - August 31, 1992

\author{
Fa-Ten Kao, Ph.D. \\ Eleanor Roosevelt Institute for Cancer Research, Inc. \\ 1899 Gaylord Street \\ Denver, CO 80206
}

August 1992

Prepared for

THE U.S. DEPARTMENT OF ENERGY AGREEMENT NO. DE-FGO2-91ER61139.AOO1

\begin{abstract}
NOTICE
This report was prepared as an account of work sponsored by the United States Government. Neither the United States nor the Department of Energy, nor any of their employees, nor any of their contractors, subcontractors, or their employees, makes any warranty, express or implied, or assumes any legal liability or responsibility for the acsuracy, completeness, or usefulness of any information, apparati2.s, product or process disclosed or represents that its use would not infringe privately-owned rights.
\end{abstract}

\section{MASTER}




\begin{abstract}
Significant progress has been made during the grant period from September 1, 1991 to August 31, 1992, in the following four areas: (1) Successful lemonstration. of regional mapping of microclones derived from microdissection libraries. (2) Successful demonstration of the feasibility of converting microclones with short inserts into yeast artificial chromosome (YAC) clones with very large inserts for high resolution physical mapping of the dissected region. (3) Successful demonstration of the usefulness of region-speciric microclones to isolate region-specific cDNA clones as candidate genes to facintate search for the crucial genes underlying genetic diseases assigned to the dissected region. (4) Successful construction of four region-specific microdissection libraries for human chromosome 2, includirng 2q35-q37, 2q33-q35, 2p23-p25 and 2p21-p23. The 2q35-q37 library has been characterized in detail. The characterization of the other three libraries is in progress. These region-specific microdissection libraries and the unique sequence microclones derived from the libraries will be valuable resources for investigators engaged in high resolution physical mapping and isolation of disease-related genes residing in these chromosomal regions.
\end{abstract}




\section{PROGRESS REPORT (September 1, 1991 - August 31, 1992)}

During the past year, we have made rapid progress towards the applications of the microdissection and microcloning techniques which we developed under the auspices of the present research grant. These include: (1) Successful demonstration of the usefulness for regional mapping of unique sequence microclones with short inserts derived from microdissection libraries. (2) Successful demonstration of the feasibility in converting unique sequence microclones with short inserts into YAC clones with very large inserts. (3) Successful demonstration of the usefulness of isolating region-specific cDNA clones from region-specific microdissection libraries. (4) Successful construction of four region-specific libraries from human chromosome 2. These studies are described in the following sections. Most of the results have been either published or in the process of publication.

(1) Successful demonstration of the usefulness for regional mapping of unique sequence microclones with short inserts derived from microdissection libraries.

Previously, we constructed a microdissection library for human chromosome 21 and obtained 700,000 microclones (1). From this library, we isolated and characterized large numbers of unique sequence microclones. The insert size in the microclones ranged from $50 \mathrm{bp}$ to $1,100 \mathrm{bp}$, with a mean of $416 \mathrm{bp}$. For the microclones with short inserts to be useful as probes, we labeled inserts from 34 unique sequence microclones and used successfully in regional mapping on chromosome 21 by Southern blot hybridization. A cell hybrid mapping panel which consists of 6 cell hybrids and divides chromosome 21 into 8 regions was obtained from Dr. David Patterson of our Institute. The inserts from microclones were amplified by PCR and labeled by primer extension. After hybridization and autoradiography, human hybridizing genomic fragments cleaved with HindIII were clearly sean in the hybrid panel. The microclones were assigned unequivocally to specific regions of chromosome 21. The mapping results are shown in Fig. 1.

Figure 1

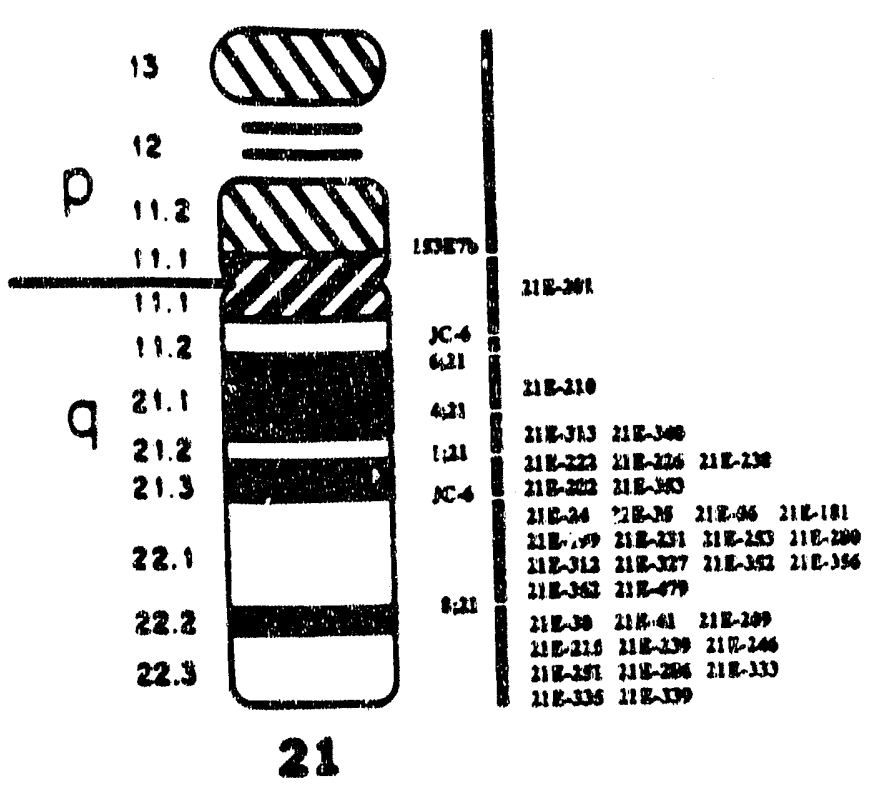


Thus, these micrnclories with short inserts can be used efficiently as probes in Southern hybridization not only for regional mapping, but also for screening other libraries as described in later sections.

From Fig. 1, uneven distribution of unique sequence microclones on chromosome 21 is evident, with the majority of clones located in the distal half of the long arm between 21q21.3 and 21qter. The number of unique sequence clones began to decrease significantly from 21q21.2 to centromere and extending to the short arm. This finding is consistent with those reported in other chromosome 21 libraries. Thus, it may be inferred that the proximal portion of the long arm of chromosome 21 contains higher proportions of repetitive sequences rather than unique sequences or genes. However, not the entire Giemsa dark band 21q21 is less represented by unique sequences. A significant portion of this dark band, including 21q21. 2 , appears to contain unique sequences as abundant as the Giemsa light band region 2 Iq22 (Fig. 1). In fact, the gene coding for the $\beta$-amyloid precursor protein is located in this dark band region 21q21,2-q21.3. Thus, one should not be tempted to generalize the composition of any Giemsa dark band in the human genome as being a region with abundant repetitive sequences. Details of these studies have been published (2).

From these results, it is clear that if there is uneven distribution of unique sequences isolated from chromosome 21 libraries, one of the most efficient ways to isolate unique sequence probes from the proximal portion of the long arm of chrornosome 21 would be by direct microdissection and cloning of this region. Such directed efforts should likely yield unique sequence probes from any underrepresented region so that sufficient YAC clones can be isolated for contig construction and physical mapping, and cDNA clones can be isolated for studying genotype/phenotype correlations of Down syndrome.

(2) Successful demonstration of the feasibility of converting microclunes with short inserts into YAC clones with very large inserts for effective coverage and high resolution physical mapping of the dissected region.

For micraclones to be most useful for molecular analysis of the dissected region, they need to be usable for screening other genomic libraries with large inserts. To this end, we demonstrated the efficient screening of a human YAC library using unique sequence microclones as labeled probes in Southern hybridization, and identified corresponding YAC clones with inserts several hundred times larger than those in the microclones.

In these experiments, we used a pooled YAC screening procedure developed by Dr. Robert Gemmill of our Institute (Genomics 10,661-665, 1991). Briefly, the human genomic YAC library of Washington University containing approximately 60,000 clones (5x genomic equivalent) was arrayed onto 3 gels, with 50 lanes per gel. Each lane contained DNAs from 384 pooled YAC clones. After pulsed-field gel electrophoresis (PFGE) and Southern transfer, the filters were hybridized to the 
pooled microclones. A pool of 3 unique sequence microclones was used in the screening. The inserts in the microclones were individually amplified by PCR, purified by low melting point gel electrophoresis, pooled to prepare labeled probes by primer extension, and hybridized to filters at $42^{\circ} \mathrm{C}$ overnight. The X-ray film was exposed for $2-9$ days at $-70^{\circ} \mathrm{C}$. After screening two-thirds of the liorary (2 filters), 5 positive YAC clones were identified, with insert sizes of $250 \mathrm{~kb}, 230 \mathrm{~kb}$ and $50 \mathrm{~kb}$ respectively. Details of these results have been published (2). Thus, it appears that the pooled YAC screening method can be used in screening YAC libraries with pooled unique sequence microclones to expand the genomic coverage in the dissected region.

(3) Successful demonstration of the usefulness of using region-specific microclones in isolating region-specific cDNA clones as candidate genes to facilitate cloning of disease-related genes assigned to the dissected chromosomal region.

In another series of studies, we demonstrated that the unique sequence microclones from microdissection libraries can be used efficiently in screening cDNA libraries to isolate region-specific expressed gene sequences from the dissected region. In these experiments, inserts from unique sequence microclones of the chromosome 21 microdissection library were individually amplified and pooled, 10 or 20 clones in a group, to prepare labeled probes. A human liver $\lambda$ gt $11 \mathrm{cDNA}$ library was used. Phages from the library were inoculated at a density of 50,000 pfu per $100 \mathrm{~mm}$ dish. One or two dishes were screened for each group of pooled microclones. Duplicate filters were prepared for each dish and used in hybridization to confirm positive signals. Positive areas detected in both duplicate filters in the primary screening were isolated and the plaques were purified by secondary and tertiary screening. The results are presented in Table 1 on Page 5. 
TABLE 1

\begin{tabular}{c|c|c}
\hline No. Clones Pooled & No. Positive Clones Per Plate & cDNA Clone Identified \\
\hline 10 & $0,0^{\mathrm{a}}$ & \\
\hline 10 & 0,0 & \\
\hline 10 & 1,0 & $21 \mathrm{E}-\mathrm{C} 1$ \\
\hline 10 & 0,0 & \\
\hline 10 & 0,0 & \\
\hline 10 & 0,0 & \\
\hline 10 & 0,0 & \\
\hline 10 & 0,0 & \\
\hline 10 & 0,0 & \\
\hline 10 & 0,0 & \\
\hline 20 & $0^{\mathrm{b}}$ & \\
\hline 20 & 0 & $21 \mathrm{E}-\mathrm{C} 2$ \\
\hline 20 & 1 & \\
\hline 20 & 0 & \\
\hline 20 & 0 & \\
\hline 20 & 0 & \\
\hline
\end{tabular}

a Representing number of positive cDNA clones isolated per plate; 2 plates screened.

b 1 plate screened.

Two cDNA clones, 21E-C1 and 21E-C2, were isolated after screening the library with 200 microclones. Preliminary characterization of the cDNA clone $21 \mathrm{E}-\mathrm{C} 1$ showed that it contains an insert of $1.5 \mathrm{~kb}$. This cDNA clone was isolated from a pool of 10 microclones and was identified as derived from microclone 21E-181. Regional mapping assigned 21E-181 to 21q21.3-q22.1. This mapping position was also confirmed by using cDNA clone $21 \mathrm{E}-\mathrm{C} 1$ as labeled probe. We partially sequenced this ¿DNA clone and presented in Appendix Fig. 1. The sequence from 5' end contains an open reading frame (ORF) of $273 \mathrm{nt}$, and the sequence from 3 ' end contains an ORF of $312 \mathrm{nt}$. The first stop codon TAA is located at nt 313 which may signal the entry into the 3' untranslated region. We searched the GenBank for homologous sequences 
and found that the sequence bears no homology with any of the previously reported DNA sequences in the GenBank, an indication of a possible new cDNA clone from chromosome 21.

The other cDNA clone, $21 \mathrm{E}-\mathrm{C} 2$, contains an insert of approximately $1 \mathrm{~kb}$. This cDNA clone was isolated from a pool of 20 microclones and was subsequently identified as derived from microclone $21 \mathrm{E}-479$, which was mapped to the same region as $21 \mathrm{E}-\mathrm{C} 1$, i.e. 21q21.3-q22.1. This mapping position was confirmed by using $21 \mathrm{E}-\mathrm{C} 2$ as probe. Partial sequencing of $21 \mathrm{E}-\mathrm{C} 2$ was carried out and the sequence is presented in Appendix Fig. 2. The sequence contains an ORF of $413 \mathrm{nt}$. The first stop codon TAA is located at nt 414 and several stop codons follow: TAA at nt 477 , TAG at nt 552, TAG at nt 573, and TGA at nt 576. Thus, this stretch of sequence with several stop codons is likely within the untranslated region at the 3 ' end of the cDNA insert. A search in the GenBank also failed to reveal any homology with reported DNA sequences in the GenBank; thus it is also a possible new cDNA clone from chromosome 21. Details of these resuits have been reported (2).

From these studies, it is clear that large numbers of region-specific cDNA clones can be isolated from region-specific microclones derived from a microdissection library. The results on the two cDNA clones isolated from chromosome 21 suggest that they could be new expressed sequences from chromosome 21. Complete sequencing and detailed characterization of these cDNA clones should provide more definitive evidence as transcribed gene sequences and may reveal their possible functions. Since the number of cloned genes on chromosome 21 is still limited, less than 20, it should be helpful to isolate more cDNA clones as candidate genes for Down syndrome and other diseases on chromosome 21, e.g. Alzheimer disease (AD), amyotrophic lateral sclerosis (ALS), leukemia, mental retardation, and at least one form of epilepsy. The present studies appear promising for efficient isolation of region-specific cDNA clones from microdissection libraries.

In addition to the direct screening procedure for isolating region-specific cDNA clones as described above, we are also developing other procedures for a more efficient isolation of cDNA clones. We are currently using the "immobilized filter hybridization" methods described by Lovett et al. (PNAS 88, 9628-9632, 1991) and by Parimoo et al. (PNAS 88, 9623-9627, 1991). In these experiments, we immobilized on filters the amplified microclone inserts from the chromosome 21 microdissection library and hybridized the filters with amplified inserts from cDNA libraries. After removing nonspecific hybrids, the hybridized CDNAs were eluted and amplified. The approach appears promising and we are currently experimenting with various hybridization and washing conditions to achieve maximal recovery of cDNA sequences. 
(4) Successful construction of four region-specific libraries of human chromosome 2.

As described in our original grant proposal, we intended to apply microdissection and microcloning techniques to human chromosome 2 as soon a.s the techniques could be successfully developed. By now, we feel that these techniques are well developed and can be successfully applied to the construction of region-specific libraries for chromosome 2 as we previcusly anticipated.

In the first series of microdissection, we aimed at the following four regions of chromosome 2 to construct region-specific libraries: 2q35-q37, 2q33-q35, 2p23-p25 and 2p21-p23, as diagramed in Fig. 2:

\section{Figure 2}

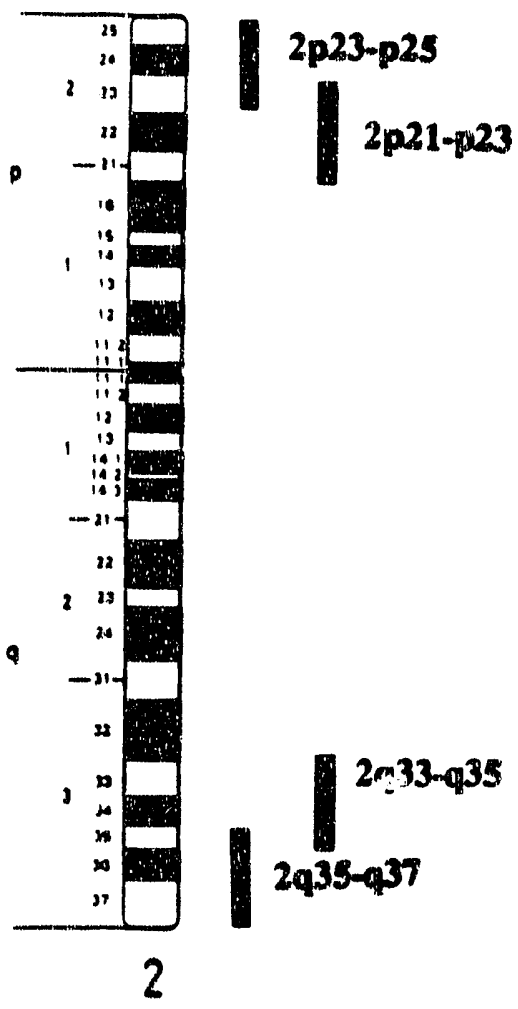

Twenty fragments were microdissected from each region. A typical microdissection is shown in Appendix Fig. 3 in which chromosome fragments from the 2q35-q37 region were dissected. The dissected fragments were treated with proteinase $\mathrm{K}$, extracted with phenol, cleaved with $\mathrm{MboI}$, and ligated to an MboI linker-adaptor. All these steps were carried out in nanoliter volumes and under the microscope. After ligation, the sample was transferred to an Eppendorf tube and amplified by PCR. The PCR products were purified and cleaved with MboI and 
cloned into pUC19. Libraries each containing 20,000-40,000 or more recombinant microclones were constructed. The 2q35-q37 library was characterized in detail. The characterization of the other three libraries is in progress.

The insert size in the microclones of the 2q35 q 37 library ranged between 50 $800 \mathrm{bp}$, with a mean of $270 \mathrm{bp}$. About 50-60\% of the microclones contained unique sequences. The library was demonstrated to derive from the region of dissection by chromosome painting using the fluorescent in situ hybridization (FISH) technique, as show $n$ in Appendix Fig. 4. In these experiments, the PCR amplified DNA from the library was labeled with biotin and hybridized in situ to human metaphase chromosomes. As can be seen from the stained chromosomes, the fluorescent label was confined to the region of dissection, i.e. the distal long arm of chromosome 2. Human chromosome 2 can be identified by its unique morphology and also by using a human chromosome 2 centromere-specific probe (which also hybridizes to the centromeres of chromosomes 18 and 20 ).

Southern blot analysis of 48 unique sequence microclones from the library showed that 54\% (26/48) are of human origin and chromosome 2 specific. In these studies, a human/CHO cell hybrid GM10826B (Cell Repository, Camden, NJ) containing a single human chromosome 2 was used. In addition, a human/mouse cell hybrid GM11022 (Cell Repository, Camden, NJ) which contained only 2q37 was also used for a more refined mapping within 2q35-q37. Four microclones showed positive hybridization with this cell hybrid, thus further assigned to the 2q37 region. Finally, these 26 unique sequence microclones were characterized for their insert size and used to identify the hybridizing genomic fragments cleaved with HindIII, as presented in Appendix Table 1. For details of these studies, please refer to (3) and (4). As demonstrated previously, these microclones will be useful in isolating corresponding YAC clones with large inserts for high resolution physical mapping, and also in screening cDNA libraries to isolate region-specific cDNA clones as candidate genes to facilitate search for the crucial genes underlying genetic diseases and specific forms of cancer assigned to the region.

Several disease-related genes and disease phenotypes have been mapped, some tentatively, to regions including 2q35-q37, e.g., oxalosis I (2q36-q37), Waardenbur syndrome, type I (2q37), alveolar rhabdomyosarcoma (2q37), Elhers-Danlos syndrome, type X (2q34-q36), cataract, Copock-like (2q33-q35), cerebrotendinosus xanthomatosis (2q33-qter), and cardiomyopathy (2q35) (ref. McKusick, J. NIH Res. 3, 143-168, 1991; Human Gene Mapping 11, 1991). The region-specific library 2q35-q37 should be particularly useful in high resolution physical mapping of this region and for studying the diseases and disease-related genes residing in this region. 


\section{REFERENCES}

(1) Kao FT, Yu J (1991) Chromosome microdissection and cloning in human genome and genetic disease analysis. Proc. Natl. Acad. Sci. USA 88, 1844-1848.

(2) Yu J, Hartz J, Xu Y, Gemmill RM, Korenberg JR, Patterson D, Kao FT (1992) Isolation, characterization and regional mapping of microclones from a human chromosome 21 microdissection library. Am. J. Hum. Genet. 51, 263-272. (A copy is enclosed)

(3) Yu J, Tong S, Yang-Feng T, Kao FT Construction and characterization of a regionspecific microdissection library from human chromosome 2q35-q37. Genomics, in press. (A copy is enclosed)

(4) Kao FT, Tong S, Yang-Feng T, Yu J (1992) Construction and characterization of region-specific microdissection libraries of human chromosome 2. Abstract presented in the 42nd Annual Meeting of the American Society of Human Genetics, Nov. 9-13, 1992, San Francisco. (A copy is enclosed) 

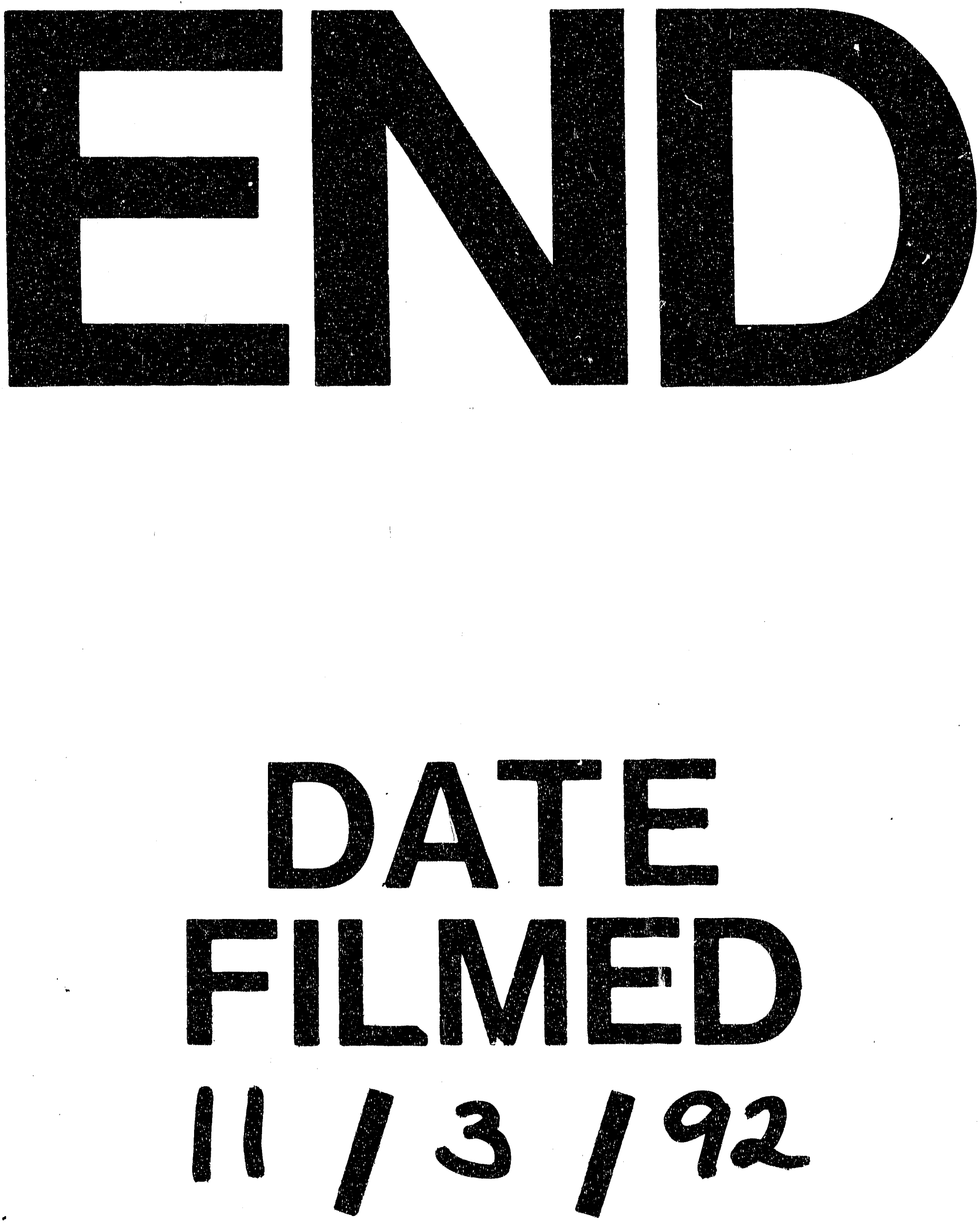

1 
